\title{
Intestinal SIRT1 Deficiency Protects Mice from Ethanol-Induced Liver Injury by Mitigating Ferroptosis
}

Zhou Zhou, ${ }^{*}$ Ting Jie Ye, ${ }^{\dagger}$ Elizabeth DeCaro, * Brian Buehler, * Zachary Stahl, * Gregory Bonavita, ${ }^{*}$ Michael Daniels, * and Min You*

From the College of Pharmacy, ${ }^{*}$ Northeast Ohio Medical University, Rootstown, Ohio; and the Department of Biology, ${ }^{\dagger}$ School of Basic Medical Science, Shanghai University of Traditional Chinese Medicine, Shanghai, China

Accepted for publication September 24, 2019.

Address correspondence to Min You, Ph.D., Department of Pharmaceutical Sciences, College of Pharmacy, Northeast Ohio Medical University, 4209 St. Rt. 44, PO Box 95, Rootstown, OH 44272. E-mail: myou@neomed.edu.

\begin{abstract}
Aberrant liver sirtuin 1 (SIRT1), a mammalian $N A D^{+}$-dependent protein deacetylase, is implicated in the pathogenesis of alcoholic liver disease (ALD). However, the role of intestinal SIRT1 in ALD is presently unknown. This study investigated the involvement of intestine-specific SIRT1 in ethanol-induced liver dysfunction in mice. Ethanol feeding studies were performed on knockout mice with intestinal-specific SIRT1 deletion [SIRT1i knockout (KO)] and flox control [wild-type (WT)] mice with a chronic-plusbinge ethanol feeding protocol. After ethanol administration, hepatic inflammation and liver injury were substantially attenuated in the SIRT1iKO mice compared with the WT mice, suggesting that intestinal SIRT1 played a detrimental role in the ethanol-induced liver injury. Mechanistically, the hepatic protective effect of intestinal SIRT1 deficiency was attributable to ameliorated dysfunctional iron metabolism, increased hepatic glutathione contents, and attenuated lipid peroxidation, along with inhibition of a panel of genes implicated in the ferroptosis process in the livers of ethanol-fed mice. This study demonstrates that ablation of intestinal SIRT1 protected mice from the ethanol-induced inflammation and liver damage. The protective effects of intestinal SIRT1 deficiency are mediated, at least partially, by mitigating hepatic ferroptosis. Targeting intestinal SIRT1 or dampening hepatic ferroptosis signaling may have therapeutic potential for ALD in humans. (Am J Pathol 2020, 190: 82 -92; https://doi.org/10.1016/j.ajpath.2019.09.012)
\end{abstract}

Alcoholic liver disease (ALD) is an alcohol-associated pathologic process characterized by a range of liver disorders from steatosis, steatohepatitis, hepatitis, fibrosis/ cirrhosis, to hepatocellular carcinoma and liver failure. ${ }^{1,2}$ Studies in rodents and humans have demonstrated that excessive alcohol consumption induces pathophysiological conditions in multiple organs. Ethanol-induced liver dysfunctions are driven by organ crosstalk via intestine-liver, adipose-liver, or adipose-intestine-liver axis. ${ }^{1-3}$ Thus, identifying functional signaling molecules in the ethanolmediated interorgan communications can lead to novel therapeutic strategies in treating human ALD.

Gut is one of the major metabolic organs involved in the development and progression of ethanol-induced liver damage. ${ }^{1-3}$ Alcohol intake induces liver dysfunction by breaking barrier integrity and function of small intestine and by promoting growth of intestinal pathogenic bacteria and harmful metabolites (eg, lipopolysaccharides). ${ }^{1,2}$ Ethanol exposure damages the liver by impairing fibroblast growth factor (FGF) 15/19, an ileum-derived hormone, and by disrupting FGF 15/19-mediated signaling. ${ }^{4-6}$ Altered intestinal microbiota and abnormal bile acid metabolism are also involved in the pathogenesis of ALD. ${ }^{4}$

Sirtuin 1 (SIRT1), a class III histone deacetylase, plays critical and controversial roles in intestinal inflammation and development of colitis. ${ }^{7-10}$ Intestinal SIRT1 deficiency

Supported in part by National Institute on Alcohol Abuse and Alcoholism grants R01AA015951 (M.Y.), R01AA013623 (M.Y.), and P50AA024333 (PI: Laura Nagy).

Z.Z. and T.J.Y. contributed equally to this work.

Disclosures: None declared. 
leads to abnormal gut microbiota, reduces inflammation, and protects mice from colitis. ${ }^{7}$ On the contrary, intestinal SIRT1 deficiency in mice increases concentrations of fecal bile acid, which in turn alter gut microbial composition, enhance intestinal inflammation, and increase susceptibility to colitis. ${ }^{8,9}$ More important, intestine-specific SIRT1 regulates the homeostasis of extra-intestinal organs (eg, liver). For instance, intestine-specific ablation of SIRT1 alters systemic homeostasis of bile acid by increasing biosynthesis of hepatic bile acid and by attenuating hepatic accumulation of bile acids, and thus, protects mice from bile acid-induced liver damage. ${ }^{9}$

Hepatic SIRT1 has been identified as a protective player against alcoholic steatohepatitis in rodents and humans. ${ }^{11,12}$ Ablation of SIRT1 in hepatocytes exacerbated the development of steatosis, inflammation, and fibrosis in mice after ethanol consumption. ${ }^{12}$ Treatment with a SIRT1 activator, resveratrol, impeded alcoholic fatty liver disease in mice experiments. ${ }^{13}$ Nevertheless, it is still unknown whether intestinal SIRT1 acts as a coordinator in the gut-liver crosstalk and contributes to the development and progression of alcoholic steatohepatitis.

In this study, with the use of an intestine-specific SIRT1 knockout mouse model (SIRT1iKO) and a chronic-plusbinge ethanol feeding protocol, ${ }^{14}$ we investigated the roles of intestinal SIRT1 in ethanol-induced liver dysfunction. We demonstrate that intestinal deficiency of SIRT1 protects mice from the ethanol-induced liver damage by attenuating a novel form of iron-dependent cell death, ferroptosis.

\section{Materials and Methods}

\section{Reagents}

Antibodies of lipocalin 2 (LCN2) and serum amyloid A1 (SAA1) were purchased from Abcam (Cambridge, MA). SIRT1 antibody was purchased from MilliporeSigma (Cleveland, $\mathrm{OH}$ ). Antibodies of glyceraldehyde-3-phosphate dehydrogenase (GAPDH) and $\beta$-actin were purchased from Cell Signal Technology (Beverly, MA).

\section{Animal Model of Chronic-Plus-Binge Alcohol Feeding}

The intestinal-specific SIRT1 KO mice (SIRT1iKO) were kindly gifted by Dr. Xiaoling Li (National Institute of Environmental Health Sciences/NIH, Research Triangle Park, NC). These SIRT1iKO mice were produced by crossing mice carrying a SIRT1 exon 4 floxed allele

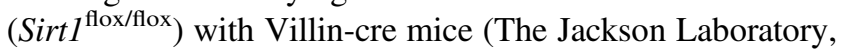
Bar Harbor, ME) in a C57BL/6 background. ${ }^{8,9}$

Our study used 10- to 12-week-old female SIRT1iKO and female flox control [wild-type (WT)] mice. These female mice were subjected to a chronic plus a single binge ethanol feeding protocol [referred as the National Institute on Alcohol Abuse and Alcoholism (NIAAA) model]. ${ }^{14}$ Mice were divided into four dietary groups: i) WT control; ii) WT plus ethanol (identical to the control diet but with $5 \% \mathrm{v} / \mathrm{w}$ ethanol added); iii) SIRT1iKO control; iv) SIRT1iKO plus ethanol (SIRT1iKO + E). All female mice were first fed a liquid control diet (Lieber-DeCarli formulation; Bioserv, Flemington, NJ) for 5 days. Ethanol groups were then fed a liquid diet containing $5 \% \mathrm{v} / \mathrm{w}$ ethanol for 10 days, whereas control mice were pair-fed to their ethanolfed counterparts for 10 days. At day 11, the ethanol groups were given a single oral gavage of ethanol $(5 \mathrm{~g} / \mathrm{kg}$ body weight, $31.25 \%$ ethanol), whereas WT or SIRT1iKO control mice were given an isocaloric gavage of dextrin maltose. No significant differences were found in food intake among the WT and SIRT1iKO mice groups fed with or without alcohol during the ethanol feeding period. Mice blood and tissues were collected after euthanasia. All animal experiments were approved by the Institutional Animal Care and Use Committee at Northeast Ohio Medical University.

\section{Serum and Liver Tissue Analysis}

Serum analyses were performed with a SpectraMax i3x microplate reader (Molecular Devices, Sunnyvale, CA). Serum alanine transaminase (ALT) and aspartate aminotransferase (AST) levels were measured with ALT and AST assay kits (BioVision, Milpitas, CA). Serum lipocalin 2 (LCN2) levels were measured by using a Lipocalin-2 Mouse ELISA (enzyme-linked immunosorbent assay) Kit (R\&D Systems, Minneapolis, MN). Serum amyloid A 1 (SAA1) levels were measured by using a SAA1 Mouse ELISA Kit (R\&D Systems). Liver cholesterol and triglyceride levels were measured as described previously. 5,6,12

\section{Histologic Analysis}

Liver or intestine tissues were fixed in $10 \%$ formalin and embedded in paraffin. Sections of $4-\mu \mathrm{m}$ thickness were prepared before staining. ${ }^{5,6}$ Hematoxylin and eosin staining and Prussian Blue staining were performed with a commercial staining kit (Sigma, St. Louis, MO). ${ }^{15}$ Immunohistochemistry staining of myeloperoxidase (MPO) was performed with a primary MPO antibody (Biocare Medical, Pacheco, CA) and a horseradish peroxidase-based secondary detection kit (Vector Laboratories, Burlingame, CA).

\section{MP0 Activity Assay}

Liver MPO activity was determined by using assay kits (Biovision, Milpitas, CA) as described previously.,

\section{Lipid Peroxidation Assay}

Liver concentrations of malondialdehyde (MDA), one of the end products of lipid peroxidation, was measured with a lipid peroxidation assay kit (Cayman, Ann Arbor, MI). ${ }^{5,6,12}$ 


\section{Iron Assay}

Iron concentrations were measured with an Iron Assay Kit (ab83366; Abcam) according to the manufacturer's instructions. ${ }^{5,15}$

\section{GSH and $\mathrm{NADP}^{+}$and NADPH Assays}

Hepatic glutathione (GSH) level was assessed with a GSH luminesce assay kit (Promega, Madison, WI) according to the manufacturer's instructions. ${ }^{15}$ Hepatic nicotinamide adenine dinucleotide phosphate (NADP) ${ }^{+}$and NADPH concentrations were assessed with a NADPH colorimetric assay kit (Abcam).

\section{GPX Activity Assay}

Liver glutathione peroxidase (GPX) enzyme activity was analyzed with a commercialized colorimetric assay kit (Biovision). ${ }^{15}$

\section{RNA Isolation and Quantitative RT-PCR}

Total RNA was purified from tissues with the use of TRIzol (Invitrogen, Waltham, MA) ${ }^{5,6,12}$ Levels of mRNA were determined by quantitative RT-PCR on a Bio-Rad thermocycler systems (Bio-Rad Laboratories, Hercules, CA). Relative mRNA levels were calculated with the comparative cycle threshold method and were normalized to the levels of GAPDH mRNA. Primer sets were either purchased or custom designed (Table 1).

\section{Western Blot Analysis}

Proteins were extracted from mouse liver or intestine tissue samples. Protein concentrations were determined with a Pierce BCA Protein Assay Kit (Thermo Scientific, Waltham, MA). Primary antibodies and secondary antibodies linked with horseradish peroxidase were used for detection as previously described. ${ }^{5,6,15}$

\section{Statistical Analysis}

Statistical significance between two groups was determined with a two-tailed $t$-test, and statistical significance between multiple groups was determined with one-way analysis of variance, followed by a Tukey post hoc test with the use of GraphPad Prism software version 8 (GraphPad Software Inc., San Diego, CA). Data were presented as means \pm SEM; $P<0.05$ was considered to be statistically significant.

\section{Results}

Ablation of Intestine-Specific SIRT1 Protects Mice from Ethanol-Induced Liver Injury

Consistent with published findings, ${ }^{8,9}$ the SIRT1iKO mice on the C57BL/6 background appeared healthy under a chow diet.
Table 1 Primer Sequence for Real-Time PCR

\begin{tabular}{|c|c|c|}
\hline Gene & Direction & Sequence \\
\hline \multirow[t]{2}{*}{ Nos2 } & $\mathrm{F}$ & 5'-GTTCTCAGCCCAACAATACAAGA-3' \\
\hline & $\mathrm{R}$ & 5'-GTGGACGGGTCGATGTCAC-3' \\
\hline \multirow[t]{2}{*}{ Gapdh } & $\mathrm{F}$ & 5'-TGACCTCAACTACATGGTCTACA-3' \\
\hline & $\mathrm{R}$ & 5'-СТTCCСАTTCTCGGCСTTG-3' \\
\hline \multirow[t]{2}{*}{ Il1 $\beta$} & $\mathrm{F}$ & $5^{\prime}-$ TCGCTCAGGGTCACAAGAAA-3' \\
\hline & $\mathrm{R}$ & $5^{\prime}$-CATCAGAGGCAAGGAGGAAAAC - 3' \\
\hline \multirow[t]{2}{*}{ Icam1 } & $\mathrm{F}$ & 5'-CAАTTTCTCATGCCGCACAG-3' \\
\hline & $\mathrm{R}$ & $5^{\prime}$-AGCTGGAAGATCGAAAGTCCG-3' \\
\hline \multirow[t]{2}{*}{ Cisd1 } & $\mathrm{F}$ & 5'-GAGAGTAACGGCCTTTTTGTGA-3' \\
\hline & $\mathrm{R}$ & 5'-GCTGTGCGAGTTGAGTGGAT-3' \\
\hline \multirow[t]{2}{*}{ Cisd2 } & $\mathrm{F}$ & $5^{\prime}$-GACAGCATCACCGGGTTCG-3' \\
\hline & $\mathrm{R}$ & $5^{\prime}$-СTCATTCACCACСTTGGGATTTT-3' \\
\hline \multirow[t]{2}{*}{ Lcn2 } & $\mathrm{F}$ & 5'-TGGCCCTGAGTGTCATGTG-3' \\
\hline & $\mathrm{R}$ & $5^{\prime}$-CTCTTGTAGCTCATAGATGGTGC-3' \\
\hline \multirow[t]{2}{*}{ Ly6g } & $\mathrm{F}$ & $5^{\prime}-$ TGCGTTGCTCTGGAGATAGA-3' \\
\hline & $\mathrm{R}$ & $5^{\prime}$-CAGAGTAGTGGGGCAGATGG-3' \\
\hline \multirow[t]{2}{*}{ Saa1 } & $\mathrm{F}$ & 5'-TTAGCTCAGTAGGTTGTGCTGCTGG-3' \\
\hline & $\mathrm{R}$ & 5'-ACAATGTTTCCCCAGAGAGCA-3' \\
\hline \multirow[t]{2}{*}{ Tnfa } & $\mathrm{F}$ & $5^{\prime}-\mathrm{AGGCTGCCCCGACTACGT-3'}$ \\
\hline & $\mathrm{R}$ & 5'-GACTTTCTCCTGGTATGAGATAGCAAA-3 \\
\hline \multirow[t]{2}{*}{ Vcam1 } & $\mathrm{F}$ & 5'-TGAACCCAAACAGAGGCAGAGT-3' \\
\hline & $\mathrm{R}$ & 5'-GGTATCCCATCACTTGAGCAGG-3' \\
\hline \multirow[t]{2}{*}{ Acsl4 } & $\mathrm{F}$ & 5'-СTCACСАТTATATTGCTGCСTGT-3' \\
\hline & $\mathrm{R}$ & 5'-тCTCTTTGCCATAGCGTTTTTCT-3' \\
\hline \multirow[t]{2}{*}{ Ncoa4 } & $\mathrm{F}$ & 5'-GAACCATCAGGACACATGGAAA-3' \\
\hline & $\mathrm{R}$ & $5^{\prime}$-GGAGCCATAGCCTTGGGT-3' \\
\hline \multirow[t]{2}{*}{ Dpp4 } & $\mathrm{F}$ & 5'-TTTAAAGAGTACCTTTCGGGTCA-3' \\
\hline & $\mathrm{R}$ & 5'-CAAGAGAACAAACAGTCGGTCA-3' \\
\hline \multirow[t]{2}{*}{ Rpl8 } & $\mathrm{F}$ & 5'-AAGGCGCGGGTTCTGTTTT-3' \\
\hline & $\mathrm{R}$ & 5'-GCTCTGTCCGCTTCTTGAATC-3' \\
\hline \multirow[t]{2}{*}{ Cars } & $\mathrm{F}$ & 5'-CATTCTGAGCATTAGTGACGAGG-3' \\
\hline & $\mathrm{R}$ & $5^{\prime}-\mathrm{CTGCCTGAACACGTCCACAT-3^{ \prime }}$ \\
\hline \multirow[t]{2}{*}{ Got1 } & $\mathrm{F}$ & 5'-GCGCCTCCATCAGTCTTTG-3' \\
\hline & $\mathrm{R}$ & 5'-АTTCATCTGTGCGGTACGCTC-3' \\
\hline \multirow[t]{2}{*}{ Tp53 } & $\mathrm{F}$ & 5'-GCGTAAACGCTTCGAGATGTT-3' \\
\hline & $\mathrm{R}$ & 5'-TTTTTATGGCGGGAAGTAGACTG-3' \\
\hline \multirow[t]{2}{*}{ Acsf2 } & $\mathrm{F}$ & 5'-CTTCGGGAGGCTGTGTATCG-3' \\
\hline & $\mathrm{R}$ & $5^{\prime}$-CACCATTCCAGAACTGAGAGC- $3^{\prime}$ \\
\hline \multirow[t]{2}{*}{ Ireb2 } & $\mathrm{F}$ & 5'-TTСТGCСТTAСТСААТАCGGGT-3' \\
\hline & $\mathrm{R}$ & 5'-AGGGCACTTCAACATTGCTCT-3' \\
\hline \multirow[t]{2}{*}{ Atp $5 G 3$} & $\mathrm{~F}$ & 5'-CTGCATCAGTGTTATCTCGGC-3' \\
\hline & $\mathrm{R}$ & 5'-CACCAGAACCAGCAACTCCTA-3' \\
\hline \multirow[t]{2}{*}{ Chac1 } & $\mathrm{F}$ & 5'-CTGTGGATTTTCGGGTACGG-3' \\
\hline & $\mathrm{R}$ & $5^{\prime}$-ССССTATGGAAGGTGTCTCC-3' \\
\hline \multirow[t]{2}{*}{ Gls2 } & $\mathrm{F}$ & 5'-GGTCTGCGCTATAACAAACTCT-3' \\
\hline & $\mathrm{R}$ & $5^{\prime}$-CATGACACTGCCTGACTCACA-3' \\
\hline Slc1a5 & $\mathrm{F}$ & 5'-CATCAACGACTCTGTTGTAGACC-3' \\
\hline & $\mathrm{R}$ & $5^{\prime}$-CGCTGGATACAGGATTGCGG-3' \\
\hline
\end{tabular}

$F$, forward; $R$, reverse.

Western blotting analyses confirmed the removal of intestinespecific SIRT1 protein in mice (Figure 1A and Supplemental Figure S1A). The protein levels of hepatic SIRT1 in SIRT1iKO mice was normal (Supplemental Figure S1, B and C). Hematoxylin and eosin histologic analysis revealed that removal of intestinal SIRT1 in mice did not alter intestinal morphologic structure (Supplemental Figure S1D)., 
A

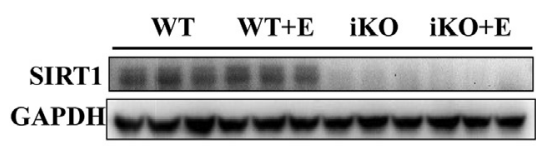

D

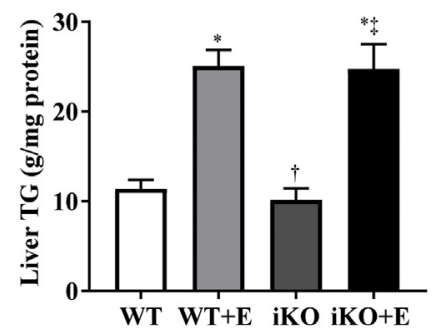

B

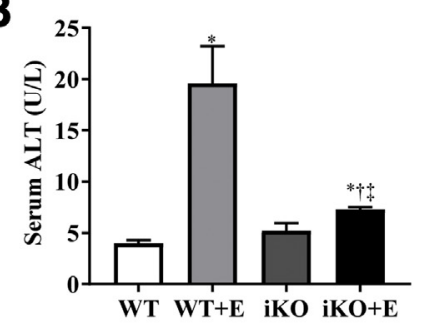

E

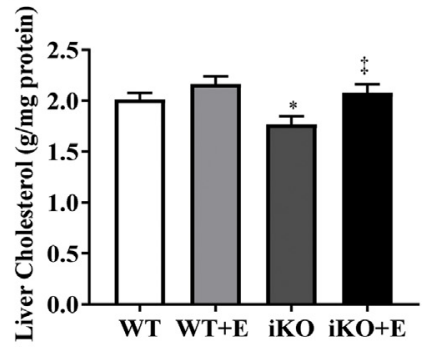

C

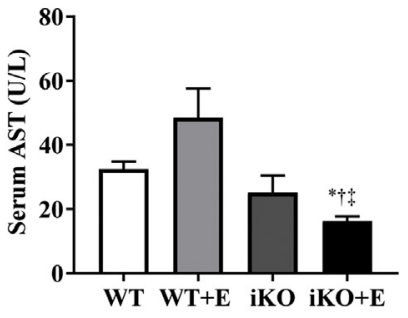

$\mathbf{F}$

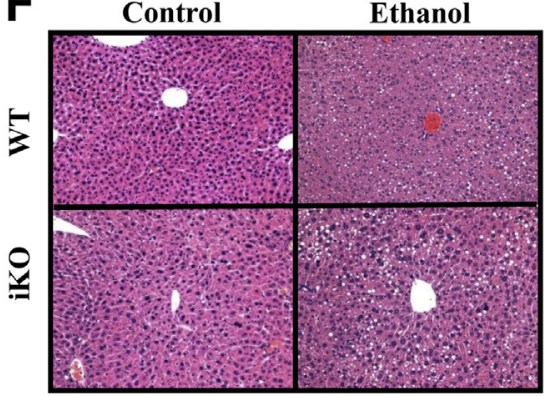

Figure 1 Intestinal sirtuin 1 (SIRT1) deficiency protects mice from ethanol (E)-induced liver injury. Female SIRT1 intestinal-specific knockout (Sirt1iK0) and wild-type (WT) mice were fed an ethanol-containing diet for 10 days, followed by single gavage of ethanol. Sirt1iK0 and WT control mice were fed control diets without ethanol for 10 days, followed by a single gavage of maltose. A: Western blot analysis of intestinal SIRT1. B: Serum alanine transaminase (ALT). C: Serum aspartate aminotransferase (AST). D: Liver triglycerides (TGS). E: Liver cholesterol. F: Hematoxylin and eosin of liver sections. Data are expressed as means \pm SEM. $n=4$ to 9 mice. ${ }^{*} P<0.05$ versus pair-fed WT mice; ${ }^{\dagger} P<0.05$ versus ethanol-fed WT mice; ${ }^{\ddagger} P<0.05$ versus intestinal SIRT1 knockout $($ SIRT1iK0) mice. Original magnification, $\times 200$. GAPDH, glyceraldehyde-3-phosphate dehydrogenase.

The involvement of intestinal SIRT1 in ethanol-induced liver damage was studied by challenging female SIRT1iKO and WT mice with ethanol. ${ }^{14}$ In comparison with the WT controls, serum ALT levels were increased by approximately fivefold in the ethanol-fed SIRT1iKO mice (Figure 1B). However, the elevation of serum ALT associated with ethanol feeding in the SIRT1iKO mice was attenuated to nearly the levels of WT control mice (Figure 1B). Concordantly, the levels of serum AST in the ethanol-fed SIRT1iKO mice were reduced to lower than that in WT mice fed with or without ethanol (Figure 1C).

Hepatic triglyceride concentrations were increased in ethanol-fed WT and ethanol-fed SIRT1iKO mice to the same extent as WT control mice (Figure 1D). Hepatic cholesterol concentrations were also similar in both ethanol-fed WT and ethanol-fed SIRT1iKO mice (Figure 1E). Liver histologic analysis confirmed similar steatosis in both WT and SIRT1iKO mice after ethanol consumption, suggesting that ethanol-induced hepatic lipid accumulation in mice may be independent of intestine-specific SIRT1 (Figure 1F). Taken together, these results demonstrated that intestinal SIRT1 deficiency ameliorated the ethanol-induced liver injury without significant alterations in steatosis in mice.

\section{Intestinal SIRT1 Deficiency Alleviates Inflammation in Ethanol-Fed Mice}

SIRT1 is a master repressor of inflammation in multiple organs, including liver and small intestine. ${ }^{7-13}$ It was investigated whether intestinal SIRT1 deficiency had any impacts on the ethanol-induced hepatic and intestinal inflammatory responses in mice.

MPO activity was robustly increased in the livers of ethanol-fed WT mice compared with WT controls (Figure 2A). Ethanol feeding to SIRT1iKO mice decreased the hepatic MPO activity to the levels of WT control mice (Figure 2A). Although $\mathrm{MPO}^{+}$neutrophils infiltrated the livers of ethanol-fed WT mice significantly higher than WT controls, the neutrophil infiltration was greatly diminished in the livers of ethanol-fed SIRT1iKO mice compared with ethanol-fed WT mice (Figure 2B). Ethanol-fed SIRT1iKO mice also exhibited the lowest mRNA levels of hepatic neutrophil marker lymphocyte antigen 6 complex, locus $\mathrm{G}$ (Ly6G) among all groups (Figure 2C). It is important to note that the hepatic Ly6G mRNA levels were higher in SIRT1iKO mice on a control diet than WT control mice (Figure 2C).

The mRNAs of hepatic tumor necrosis factor- $\alpha$ (TNF- $\alpha$ ), inducible nitric oxide synthase (iNOS), IL- $1 \beta$, vascular cell adhesion molecule (VCAM)-1, and intercellular adhesion molecule (ICAM)-1 were higher in the ethanol-fed WT mice than in the WT controls (Figure 2D). However, mRNAs for these inflammatory cytokines were attenuated in the livers of ethanol-fed SIRT1iKO mice compared with WT control or ethanol-fed WT mice (Figure 2D). In comparison with WT controls, ethanol feeding to WT mice markedly increased mRNAs of intestinal iNOS and TNF- $\alpha$ (Figure 2E). The mRNA levels of intestinal inducible nitric 
A

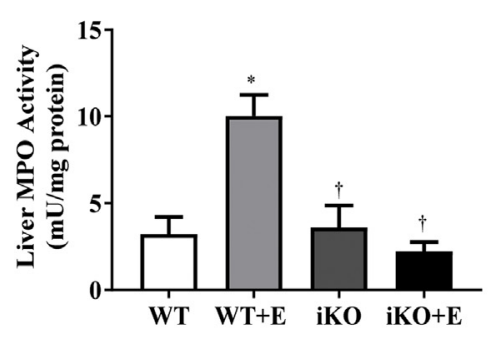

B

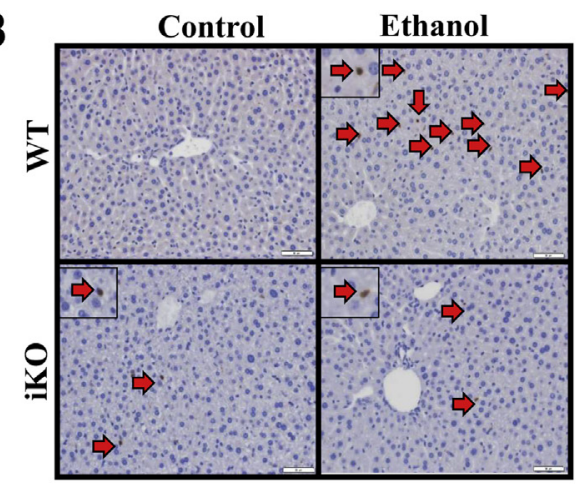

$\mathbf{E}$
C

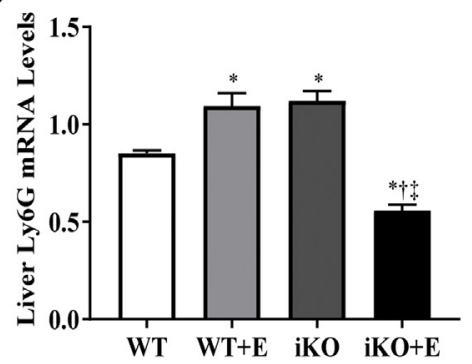

D

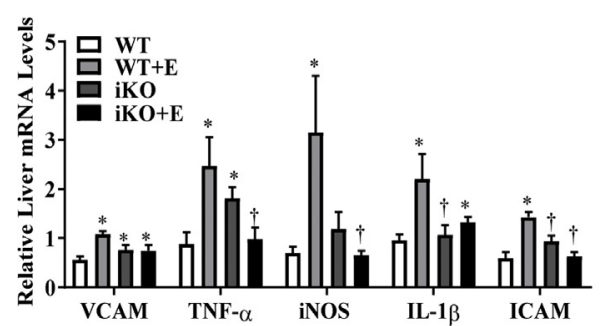

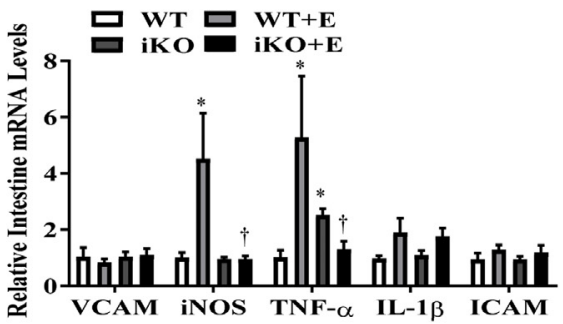

Figure 2 Intestinal sirtuin 1 (SIRT1) deficiency ameliorates hepatic inflammation in ethanol (E)-fed mice. Mice were fed as described in Figure 1. A: Hepatic myeloperoxidase (MP0) activity. B: Liver MP0 staining of liver sections. Arrows indicate MPO-positive cell staining. Insets magnify local staining details. C: Relative mRNA levels of Ly6G. D and E: Relative mRNA levels of hepatic (D) and intestinal (E) vascular cell adhesion molecule (VCAM), tumor necrosis factor (TNF)- $\alpha$, inducible nitric oxide synthase (iNOS), IL-1 $\beta$, and intercellular adhesion molecule (ICAM). Data are expressed as means \pm SEM. $n=4$ to 9 mice. ${ }^{*} P<0.05$ versus pair-fed wild-type (WT) mice; ${ }^{\dagger} P<0.05$ versus ethanol-fed WT mice; ${ }^{\ddagger} p<0.05$ versus intestinal SIRT1 knockout (SIRT1ik0) mice. Original magnification: $\times 200$ (main images); $\times 400$ (insets).

oxide synthase and tumor necrosis factor- $\alpha$ were diminished in the SIRT1iKO mice after the ethanol feeding (Figure 2E). However, the mRNAs for intestinal VCAM-1 and IL-1 $\beta$ and ICAM-1 were not altered by the ethanol feeding to SIRT1iKO mice in contrast to ethanol-fed WT mice (Figure 2E). These results demonstrated that SIRT1iKO mice were partially resistant to the ethanol-induced hepatic and intestinal inflammation, particularly hepatic neutrophilic inflammation.

\section{Intestinal SIRT1 Deficiency Blocks the Ability of Ethanol to Induce LCN2 and SAA1 in Mice}

LCN2 and SAA1 are two major players in inflammation and tissue injury, especially in ethanol-induced liver damage. $^{12,16-20}$ Thus, we hypothesized that LCN2 and SAA1 might be involved in the diminished inflammatory process and hepatic improvement in the ethanol-fed SIRT1iKO mice.

The serum levels of LCN2 and SAA1 were markedly increased in the ethanol-fed WT mice compared with the WT controls (Figure 3, A and B). SIRT1iKO mice on a control diet also displayed significantly higher serum concentrations of LCN2 and SAA1 than the WT controls (Figure 3, A and B). Strikingly, the ethanol feeding to SIRT1iKO mice reduced serum LCN2 and SAA1 to nearly the levels of WT control mice (Figure 3, A and B).
The mRNA and protein expression levels of hepatic and intestinal LCN2 and SAA1 were examined in these mice. In comparison with WT control mice, mRNA and protein levels of hepatic and intestinal LCN2 and SAA1 were elevated in WT mice after the ethanol administration (Figure 3, C-F, and Supplemental Figure S2, A-D). In line with lower circulating levels of LCN2 and SAA1, gene and protein expression levels of hepatic and intestinal LCN2 and SAA1 were reduced by feeding ethanol to SIRT1iKO mice compared with WT or ethanol-fed WT mice (Figure 3, C-F, and Supplemental Figure S2, A-D). Taken together, these results showed that intestinal SIRT1 deficiency blocked the ability of ethanol to induce gene and protein expression and circulation of hepatic and intestinal LCN2 and SAA1 in mice.

\section{Intestinal SIRT1 Deficiency in Mice Ameliorates the Ethanol-Induced Iron Metabolism Dysfunction}

Iron metabolism dysfunction is implicated in the pathogenesis of ALD. To dissect mechanisms underlying the protective effects of SIRT1iKO mice, hepatic iron contents were measured.

Consistent with our recent findings, hepatic concentrations of iron ( $\mathrm{Fe})$ and ferrous $\left(\mathrm{Fe}^{2+}\right)$ in ethanol-fed WT mice were significantly increased in comparison with WT control mice (Figure 4A). However, intestinal SIRT1 

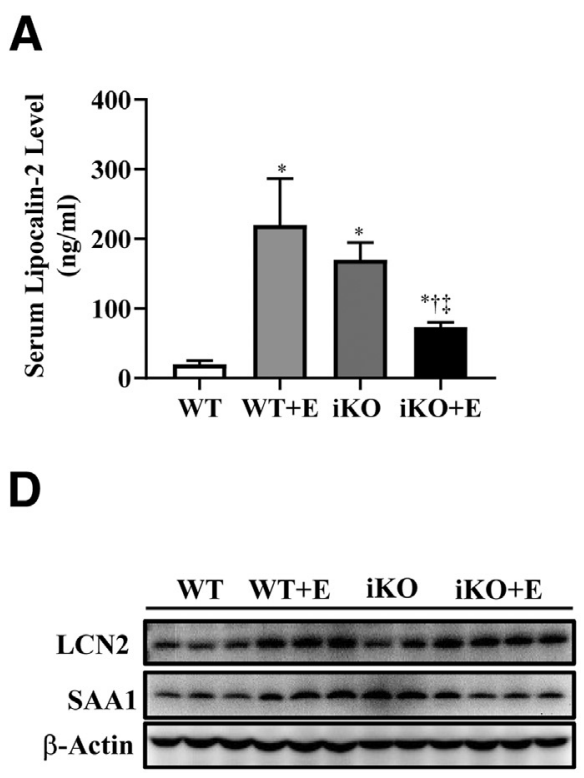

B

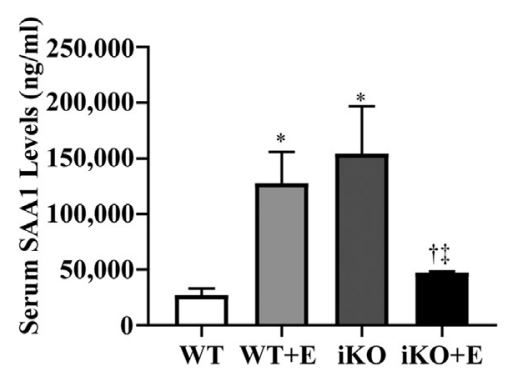

E
C

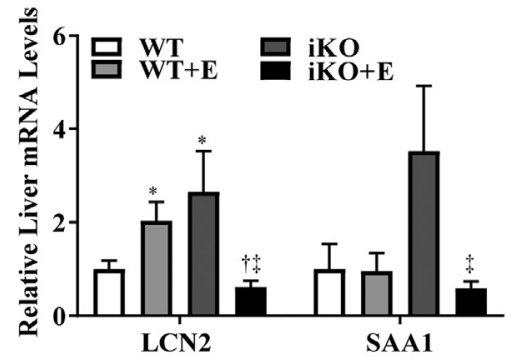

$\mathbf{F}$

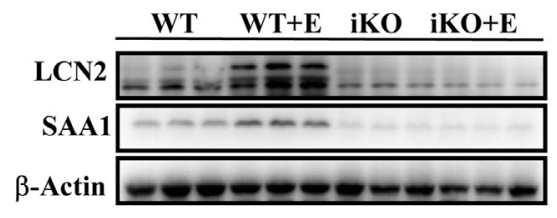

Figure 3 Intestinal sirtuin 1 (SIRT1) deficiency reduces circulating levels of lipocalin-2 (LCN2) and serum amyloid A1 (SAA1) in the ethanol (E)administrated mice. Mice were fed as described in Figure 1. A: Serum levels of LCN2. B: Serum levels of SAA1. C: Relative mRNA levels of hepatic LCN2 and SAA1. D: Western blot analysis of hepatic LCN2 and SAA1. E: Relative mRNA levels of intestinal LCN2 and SAA1. F: Western blot analysis of intestinal LCN2 and SAA1. Data are expressed as means \pm SEM. $n=4$ to 9 mice. ${ }^{*} P<0.05$ versus pair-fed wild-type (WT) mice; ${ }^{\dagger} P<0.05$ versus ethanol-fed WT mice; ${ }^{\ddagger} P<0.05$ versus intestinal SIRT1 knockout (SIRT1iK0) mice.

deficiency normalized the ethanol-mediated elevation of hepatic iron $(\mathrm{Fe})$ and ferrous $\left(\mathrm{Fe}^{2+}\right)$ levels to nearly that of WT control levels (Figure 4A). Consistently, Prussian blue staining showed that ethanol feeding to WT mice caused a prominent hepatic iron staining compared with WT controls, whereas less iron staining was spotted in ethanol-fed SIRT1iKO mice (Figure 4B). The hepatic iron (Fe) was slightly increased in the SIRT1iKO mice fed a control diet compared with WT control mice, although without reaching statistical significance (Figure 4, A and B). Intestinal SIRT1 deficiency did not alter intestinal iron concentrations in mice fed with or without ethanol (Supplemental Figure S2E).

The mRNA levels of CDGSH iron sulfur domain (CISD) 1 and CISD2, two redox active iron-sulfur (2Fe-2S) proteins involved in the ethanol-induced abnormality of iron metabolism, were next analyzed. ${ }^{5}$ Although ethanol feeding to WT mice did not alter hepatic CISD1 mRNA abundance compared with WT control mice, ethanol-fed SIRT1iKO mice exhibited the lowest mRNA levels of hepatic CISD1 in comparison with the other three groups (Figure 4C). Although hepatic CISD2 mRNA levels were significantly higher in the ethanol-fed WT mice, ethanol feeding to SIRT1iKO mice reduced hepatic CISD2 mRNA levels to nearly that of the WT control levels (Figure 4C). Similarly, intestinal CISD1 and CISD2 mRNA levels were the lowest in the ethanol-fed SIRT1iKO mice compared with their respective other three groups (Figure 4D). Taken together, the results demonstrated that intestinal SIRT1 deficiency ameliorated the ethanol-induced iron metabolism dysfunction by attenuating accumulation of hepatic iron in mice.

\section{Intestinal SIRT1 Deficiency Inhibits Hepatic Ferroptosis in Ethanol-Administrated Mice}

Ferroptosis is a new form of iron-dependent programed cell death characterized by the formation of lethal lipid peroxidation. ${ }^{21,22}$ In liver, iron is vital in ferroptosis. ${ }^{21,22}$ Therefore, we hypothesized that attenuated hepatic accumulation of iron in the ethanol-fed SIRT1iKO mice might mitigate ferroptosis and, consequently, reduce inflammation and liver injury. Known biomarkers of ferroptosis, including hepatic lipid peroxidation, $\mathrm{GSH}$, the ratio of $\mathrm{NADP}^{+} / \mathrm{NADPH}$, and GPX activity were determined. ${ }^{15,21-23}$

Ethanol feeding to WT mice significantly elevated levels of hepatic MDA, a lipid peroxidation end product (Figure 5A). However, the MDA levels in ethanol-fed SIRT1iKO mice were reduced to the WT control levels (Figure 5A). In comparison with the WT controls, liver GSH contents were significantly reduced in ethanol-fed WT mice, but were slightly increased in SIRT1iKO control mice (Figure 5B). Strikingly, hepatic GSH levels were robustly increased in the ethanol-fed SIRT1iKO mice compared with all other groups (Figure 5B). Accordingly, liver $\mathrm{NADP}^{+}$/ NADPH ratio in ethanol-fed SIRT1iKO was highest compared with all other groups (Figure 5C). In comparison 

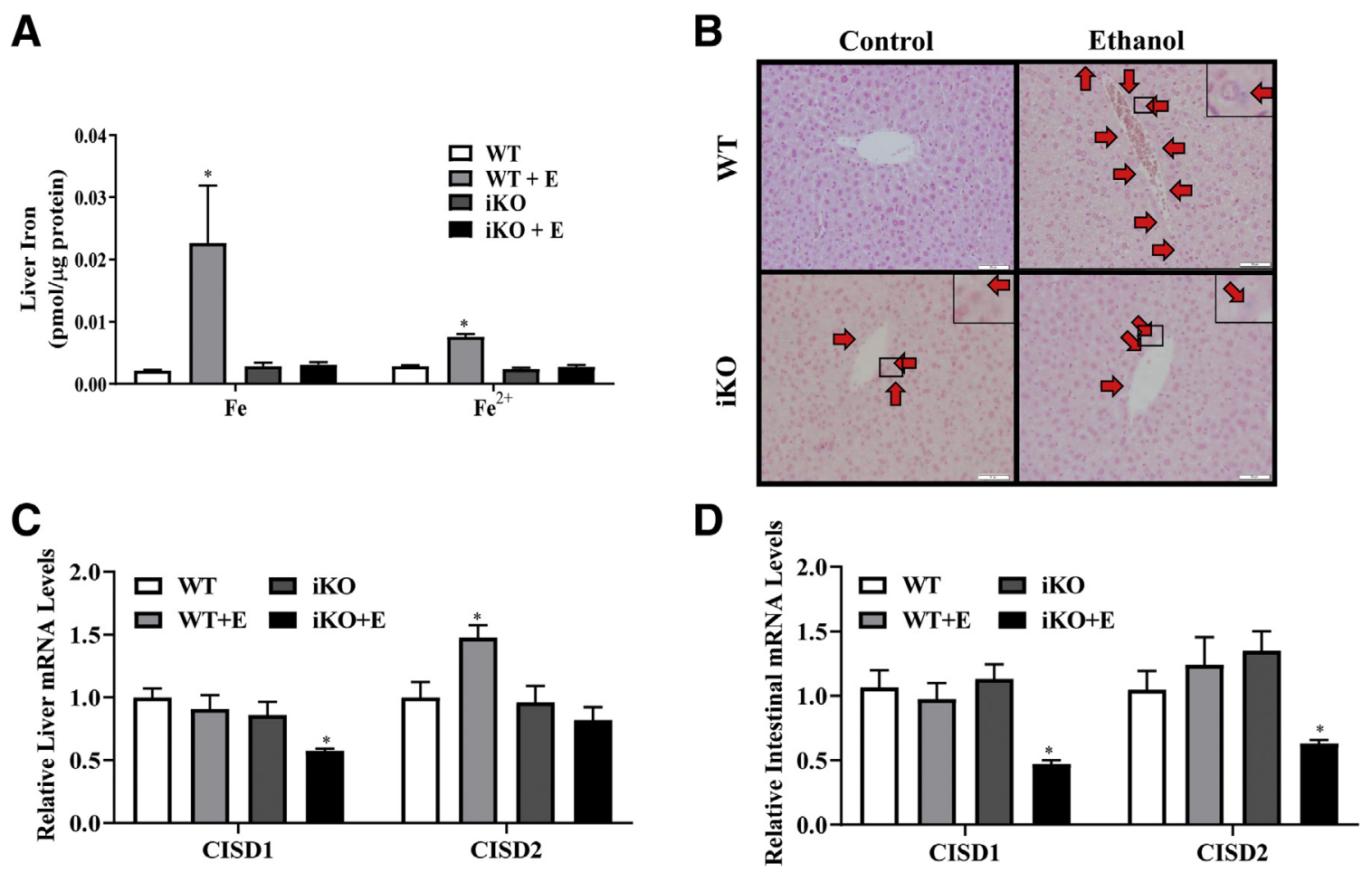

Figure 4 Intestinal sirtuin 1 (SIRT1) deficiency ameliorates aberrant iron homeostasis in ethanol (E)-fed mice. Mice were fed as described in Figure 1. A: Liver iron (Fe) and ferrous $\left(\mathrm{Fe}^{2+}\right)$ concentrations. B: Prussian Blue staining of liver sections. Arrows indicate iron-positive cell staining. Boxed areas indicate local staining details, magnified in the insets. C: Relative mRNA levels of hepatic CISD1 and CISD2. D: Relative mRNA levels of intestinal CISD1 andCISD2. Data are expressed as means \pm SEM. $n=4$ to 9 mice. ${ }^{*} P<0.05$ versus pair-fed wild-type (WT) mice. Original magnification: $\times 200$ (main images); $\times 400$ (insets). CISD, CDGSH iron sulfur domain; iKO, intestinal SIRT1 knockout.

with WT control mice, hepatic GPX activity was significant decreased by feeding ethanol to WT or SIRT1iKO mice to a similar extent, suggesting involvement of GPX4independent mechanisms in mediating effects of intestinal SIRT1 deficiency on ethanol-induced ferroptosis (Figure 5D).

To further dissect mechanisms underlying the attenuated ferroptosis in the livers of ethanol-fed SIRT1iKO mice, hepatic mRNA levels of several molecules that have been implicated in promoting ferroptosis were analyzed by quantitative real-time PCR. ${ }^{20-36}$ In comparison with WT control mice, ethanol feeding to WT mice significantly elevated the mRNAs of genes encoding Acyl-CoA synthetase family member 2, glutathione-specific gammaglutamylcyclo transferase 1, glutaminase 2, and iron-responsive element binding protein 2 (Figure 6A). Ethanol feeding also increased, but without reaching statistical significance for mRNAs encoding tumor protein 53, Cysteinyl tRNA synthetase, Solute carrier family 1 member 1 in ethanol-fed WT mice compared with WT controls (Figure 6A). Furthermore, in comparison with WT controls, mRNAs for acyl-CoA synthetase long-chain family member 4 , nuclear receptor coactivator 4, dipeptidyl-dippeptidase-4, ribosomal protein L8, glutamic-oxaloacetic transaminase 1, ATP synthase, $\mathrm{H}^{+}$ transporting, mitochondrial Fo complex subunit $\mathrm{C} 3$ (subunit 9) were either unchanged or slightly reduced in ethanol-fed
WT mice (Figure 6B). Strikingly, intestinal SIRT1 deficiency effectively attenuated mRNAs of all above molecules to levels significantly lower than those in WT control or ethanol-fed WT mice (Figure 6). Collectively, these results supported the hypothesis that intestinal SIRT1 deficiency mitigated hepatic ferroptosis and alleviated the liver damage in mice after ethanol challenge.

\section{Discussion}

The present study uncovered a previously unknown detrimental role of intestine-specific SIRT1 in ethanol-induced liver inflammation and injury. Intestinal SIRT1 deficiency drastically attenuated circulating levels of LCN2 and SAA1, two pivotal proinflammatory molecules, contributing to a diminished hepatic inflammatory response and protected mice from liver injury after ethanol consumption. Mechanistically, intestinal deficiency of SIRT1 alleviated the ethanol-induced liver injury in mice by ameliorating dysfunctional iron metabolism, enhancing GSH levels, elevating $\mathrm{NADP}^{+} / \mathrm{NADPH}$ contents, attenuating lipid peroxidation along with reduced expression of a panel of genes involved in ferroptosis in mice after ethanol challenge. Our findings suggest that the protective effect of intestinal SIRT1 deficiency against the ethanol-induced liver 
A
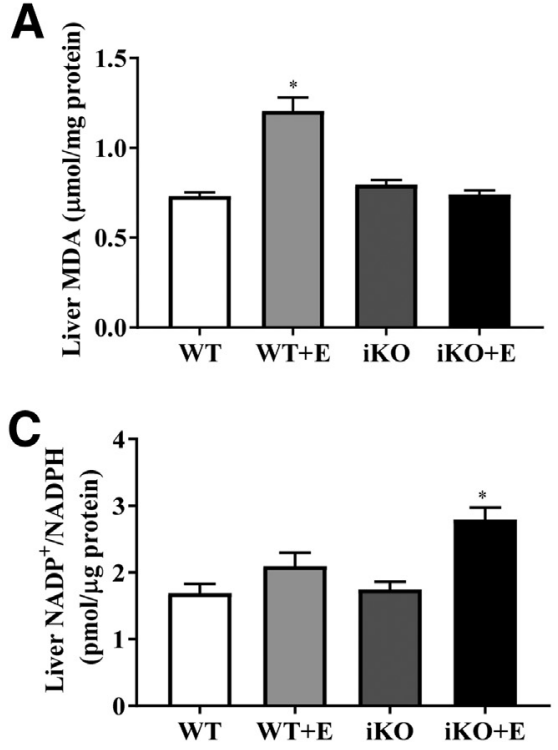

B

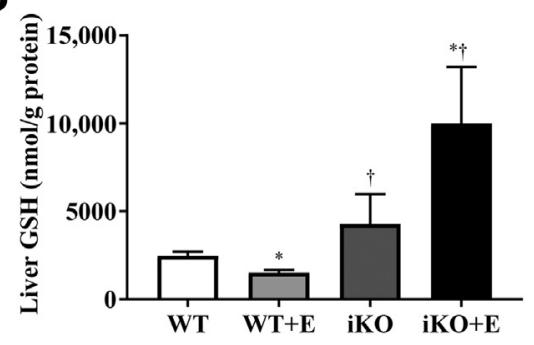

D

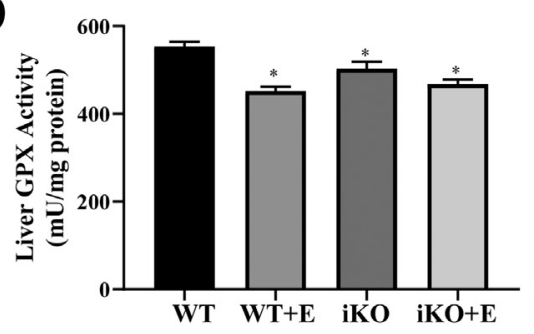

Figure 5 Intestinal sirtuin 1 (SIRT1) deficiency mitigates hepatic ferroptosis in the ethanol (E)-fed mice. Mice were fed as described in Figure 1. A: Liver malondialdehyde (MDA) contents. B: Liver glutathione (GSH) contents. C: Hepatic $\mathrm{NADP}^{+} / \mathrm{NADPH}$. D: Liver glutathione peroxidase (GPX) activity. Data are expressed as means \pm SEM. $n=4$ to 9 mice. ${ }^{*} P<0.05$ versus pair-fed wild-type (WT) mice; ${ }^{\dagger} P<0.05$ versus ethanol-fed WT mice. iKO, intestinal SIRT1 knockout. inflammation and injury is, at least partially, mediated by inhibition of hepatic ferroptosis (Figure 7).

Ethanol-fed WT mice displayed ferroptosis-based panel of biomarkers such as iron overload, lipid peroxidation, inhibition of GPX activity, induction of the ferroptosis-related gene expression, and inflammation, supporting that ethanol is capable of regulating hepatic ferroptotic machinery. However, it remains unsolved how ethanol involves in hepatic ferroptosis. The regulatory mechanisms of ferroptosis are complicated and incompletely understood. Multiple factors in iron and redox metabolism contribute to ferroptosis. For instance, small molecule erastin activates ferroptosis by depleting cysteine, inhibiting GSH synthesis, and inactivating GPX4. ${ }^{21,22}$ Iron also plays a driving role in hepatic ferroptosis. ${ }^{21,22}$ Furthermore, iron-induced ferroptosis is distinctly different from erastin-induced ferroptosis in liver. ${ }^{22}$ Thus, it is tempting to speculate that a combination of iron overload and increased lipid peroxidation along with attenuation of GSH concentration and GPX activity and induction of multiple ferroptosis-related molecules may be sufficient to steer the ferroptosis signaling cascades and ultimately induce liver injury in ethanol-fed WT mice.

Sex-specific susceptibility to ALD is well documented. $^{1-3}$ Clinical and animal models have demonstrated that females experience more extensive liver injury than males. Our preliminary studies revealed that, unlike female SIRT1iKO mice, intestinal SIRT1 deficiency had minimal effects on the liver damage in male mice in response to ethanol challenge (M. You, unpublished observation). Of interest, augmented iron concentrations were found in the livers of ethanol-fed female mice compared with ethanol-fed male mice. ${ }^{15,37}$ It is possible that the sex-specific responses in hepatic iron contents may lead to the sex differences in ferroptosis and liver damage in mice after ethanol challenge. The detailed mechanisms underlying sex differences in ferroptosis and their relationship with ethanol-induced liver damage are currently under investigation in our laboratory.

Another interesting finding of our study is the lower mRNA expression levels of CISD1 and CISD2 in the livers of ethanol-fed SIRT1iKO. CISD1 protein levels were also substantially reduced in the livers of ethanol-fed SIRT1iKO mice compared with WT control mice (M. You, unpublished observations). CISD1 and CISD2 are pivotal regulators of iron metabolism. ${ }^{38}$ Owing to [2Fe-2S] clusters, CISD1 and CISD2 can be sources of cellular free iron. ${ }^{38}$ In addition, CISD1 and CISD2 are known regulators of ferroptosis in cultured cancer cells. ${ }^{39,40}$ The ethanol-induced hepatic iron overload may be mediated through releasing [2Fe-2S] clusters in CISD1 and/or in CISD2. In this scenario, intestinal SIRT1 deficiency may attenuate hepatic CISD1 and/or CISD2 protein, which, in turn, block the ability of ethanol to release $[2 \mathrm{Fe}-2 \mathrm{~S}]$ clusters to induce iron accumulation and to generate ROS, ultimately mitigating ferroptosis.

This study suggests that LCN2-SAA1 axis may serve as a pivotal endocrine mediator in the cross-communications between gut and liver in mice after ethanol intake. The diminished LCN2-SAA1 axis-mediated signaling could be a major contributing factor to improvements observed in the livers of ethanol-fed SIRT1iKO mice. LCN2 is an important regulator in iron metabolism. ${ }^{41}$ The ability of LCN2 to regulate iron metabolism depends on whether LCN2 is bound to iron-laden siderophores. ${ }^{41}$ The ironloaded LCN2-siderophore (Holo-LCN2) serves as an iron donor and is required for delivery of iron to cells/tissues. On 

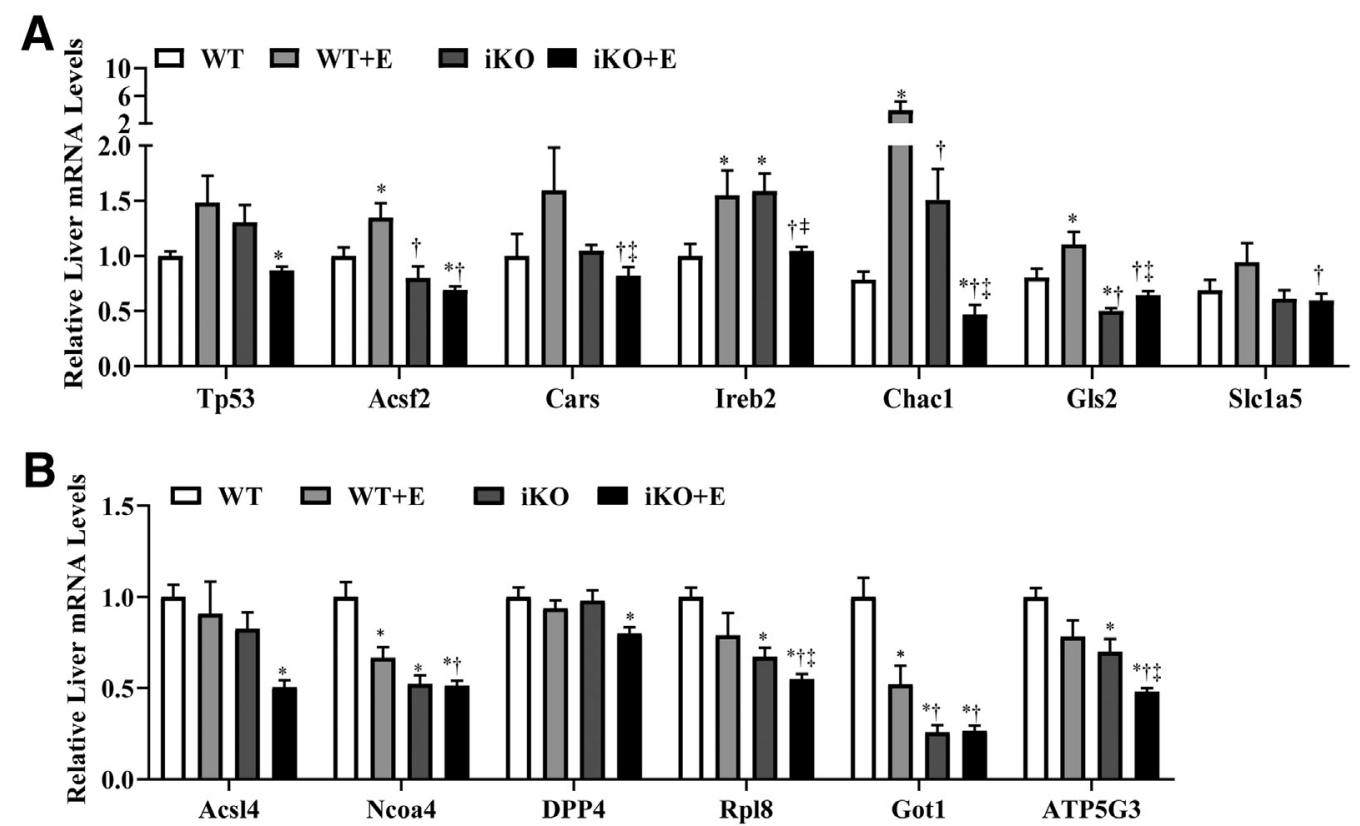

Figure 6 Intestinal sirtuin 1 (SIRT1) deficiency mitigates expression of hepatic ferroptosis-inducing genes in the ethanol (E)-fed mice. Mice were fed as described in Figure 1. A and B: Relative hepatic mRNA levels of Tp53, Acsf2, Cars, Ireb2, Chac1, Gls2, and Slc1a5 (A) and Acsl4, Ncoa4, DPP4, Rpl8, Cot1, and ATP5G3 (B). Data are expressed as means \pm SEM. $n=4$ to 9 mice. ${ }^{*} P<0.05$ versus pair-fed wild-type (WT) mice; ${ }^{\dagger} P<0.05$ versus ethanol-fed WT mice; ${ }^{\ddagger} P<0.05$ versus intestinal SIRT1 knockout (SIRT1iK0) mice.

the contrary, the LCN2-siderophore complex without iron (Apo-LCN2) functions as an iron chelator and chelates iron from cells/tissues. Ethanol-mediated hepatic iron overload may be, at least in part, mediated by increasing generation of Holo-LCN2. In this scenario, intestinal SIRT1 deficiency may reduce hepatic iron concentrations by attenuating HoloLCN2 or increasing generation of Apo-LCN2. Given that LCN2 and SAA1 are concomitantly up-regulated by ethanol, it is likely that ethanol may also trigger hepatic ferroptosis indirectly via a LCN2-SAA1 axis-mediated iron deposition. Furthermore, LCN2 drives ethanol-

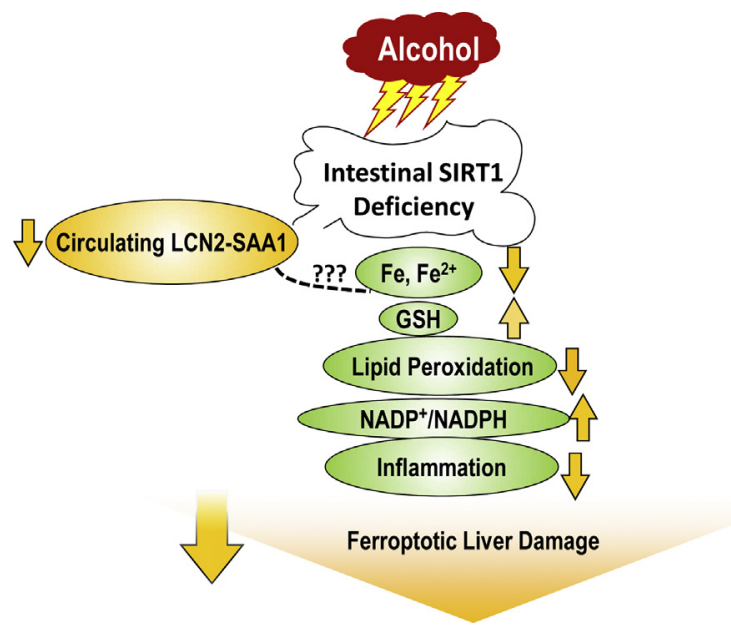

Figure 7 Schematic diagram of proposed mechanisms that underlie the protective effects of intestinal sirtuin 1 (SIRT1) deficiency against the ethanol-induced ferroptotic liver damage. Fe, iron; $\mathrm{Fe}^{2+}$, ferrous; $\mathrm{GSH}$, glutathione; LCN2, lipocalin 2; SAA1, serum amyloid A1. mediated neutrophilic inflammation in rodents and humans. ${ }^{18}$ Ferroptotic cell death is associated with neutrophil inflammation. ${ }^{42}$ Thus, the lower level of LCN2 in the ethanol-fed SIRT1iKO mice might be a response for the iron deprivation, attenuated neutrophilic information, and diminished ferropotic liver injury. Further studies are needed to investigate whether and how the LCN2-SAA1 signaling is involved in regulating iron metabolism and ferroptosis process in the context of experimental ALD.

It is intriguing that SIRT1iKO mice on a control diet exhibited higher levels of circulating LCN2 and SAA1 compared with WT control mice. Intestinal SIRT1 regulates gut microbiota and is involved in inflammatory process. ${ }^{8}$ Gut microbiota is involved in regulating LCN2 and SAA1 ${ }^{43,44}$ It is logical to speculate that the intestinal SIRT1 deficiency-induced alteration of gut microbiota may contribute to the elevated levels of circulating LCN2 and SAA1 in SIRT1iKO mice on a control diet.

In contrast to hepatic deficiency of SIRT $1,{ }^{12}$ which exacerbated experimental alcoholic steatohepatitis, deletion of intestinal SIRT1 protected mice from the ethanolinduced liver damage, highlighting the cell-/tissue-specific nature of SIRT1 functions in alcoholic steatohepatitis. SIRT1 in intestinal tract has major impacts on gut microbiome. $^{7-9}$ Alcohol intake impairs intestinal microbiota and alters gut microbiota composition, thereby contributing to liver dysfunctions. ${ }^{4}$ Intestinal SIRT1 deficiency may normalize intestinal microbiota or restore the symbiotic balance in the ethanol-fed mice and attenuate inflammation and ultimately protect mouse liver from the damaging effects of ethanol. 


\section{Conclusions}

The evidences provided in this study suggest a detrimental role of intestine-specific SIRT1 in alcohol-induced liver inflammation and injury. We have identified a novel gut-liver interaction in ALD. More important, we have demonstrated that the iron-driven ferroptosis is involved in the development and progression of alcoholic steatohepatitis. Our findings suggest that reducing iron accumulation with iron chelators or inhibiting ferroptosis in liver may be plausible therapeutic approaches to alleviate ethanolinduced liver damage in humans.

\section{Acknowledgment}

We thank Dr. Xiaoling Li (National Institute of Environmental Health Sciences/NIH) for providing SIRT1iKO mice.

\section{Supplemental Data}

Supplemental material for this article can be found at http://doi.org/10.1016/j.ajpath.2019.09.012.

\section{References}

1. Farooq MO, Bataller R: Pathogenesis and management of alcoholic liver disease. Dig Dis 2016, 34:347-355

2. Gao B, Ahmad MF, Nagy LE, Tsukamoto H: Inflammatory pathways in alcoholic steatohepatitis. J Hepatol 2019, 70:249-259

3. You M, Zhou Z, Daniels M, Jogasuria A: Endocrine adiponectinFGF15/19 axis in ethanol-induced inflammation and alcoholic liver injury. Gene Expr 2018, 18:103-113

4. Hartmann P, Hochrath K, Horvath A, Chen P, Seebauer CT, Llorente C, Wang L, Alnouti Y, Fouts DE, Stärkel P, Loomba R, Coulter S, Liddle C, Yu RT, Ling L, Rossi SJ, DePaoli AM, Downes M, Evans RM, Brenner DA, Schnabl B: Modulation of the intestinal bile acid/farnesoid $\mathrm{X}$ receptor/fibroblast growth factor 15 axis improves alcoholic liver disease in mice. Hepatology 2018, 67: $2150-2166$

5. Hu X, Jogasuria A, Wang J, Kim C, Han Y, Shen H, Wu J, You M: MitoNEET deficiency alleviates experimental alcoholic steatohepatitis in mice by stimulating endocrine adiponectin-Fgf15 axis. J Biol Chem 2016, 291:22482-22495

6. Wang J, Kim C, Jogasuria A, Han Y, Hu X, Wu J, Shen H, Chrast R, Finck BN, You M: Myeloid cell-specific lipin-1 deficiency stimulates endocrine adiponectin-FGF15 axis and ameliorates ethanol-induced liver injury in mice. Sci Rep 2016, 6:34117

7. Lo Sasso G, Ryu D, Mouchiroud L, Fernando SC, Anderson CL, Katsyuba E, Piersigilli A, Hottiger MO, Schoonjans K, Auwerx J: Loss of Sirt function improves intestinal anti-bacterial defense and protects from colitis-induced colorectal cancer. PLoS One 2014, 9: e102495

8. Wellman AS, Metukuri MR, Kazgan N, Xu X, Xu Q, Ren NSX, Czopik A, Shanahan MT, Kang A, Chen W, Azcarate-Peril MA, Gulati AS, Fargo DC, Guarente L, Li X: Intestinal epithelial Sirtuin 1 regulates intestinal inflammation during aging in mice by altering the intestinal microbiota. Gastroenterology 2017, 153:772-786

9. Kazgan N, Metukuri MR, Purushotham A, Lu J, Rao A, Lee S, PrattHyatt M, Lickteig A, Csanaky IL, Zhao Y, Dawson PA, Li X:
Intestine-specific deletion of SIRT1 in mice impairs DCoH2-HNF1alpha-FXR signaling and alters systemic bile acid homeostasis. Gastroenterology 2014, 146:1006-1016

10. Wang Y, Shi X, Qi J, Li X, Uray K, Guan X: SIRT1 inhibits the mouse intestinal motility and epithelial proliferation. Am J Physiol Gastrointest Liver Physiol 2012, 302:G207-G217

11. You M, Jogasuria A, Taylor C, Wu J: Sirtuin 1 signaling and alcoholic fatty liver disease. Hepatobiliary Surg Nutr 2015, 4:88-100

12. Yin H, Hu M, Liang X, Ajmo JM, Li X, Bataller R, Odena G, Stevens SM Jr, You M: Deletion of SIRT1 from hepatocytes in mice disrupts lipin-1 signaling and aggravates alcoholic fatty liver. Gastroenterology 2014, 146:801-811

13. Ajmo JM, Liang X, Rogers CQ, Pennock B, You M: Resveratrol alleviates alcoholic fatty liver in mice. Am J Physiol Gastrointest Liver Physiol 2008, 295:G833-G842

14. Bertola A, Mathews S, Ki SH, Wang H, Gao B: Mouse model of chronic and binge ethanol feeding (the NIAAA model). Nat Protoc 2013, 8:627-637

15. Zhou Z, Ye TJ, Bonavita G, Daniels M, Kainrad N, Jogasuria A, You M: Adipose-specific lipin-1 overexpression renders hepatic ferroptosis and exacerbates alcoholic steatohepatitis in mice. Hepatol Commun 2019, 3:656-669

16. Kong B, Zhang M, Huang M, Rizzolo D, Armstrong LE, Schumacher JD, Chow MD, Lee YH, Guo GL: FXR deficiency alters bile acid pool composition and exacerbates chronic alcohol induced liver injury. Dig Liver Dis 2019, 51:570-576

17. Cai Y, Jogasuria A, Yin H, Xu MJ, Hu X, Wang J, Kim C, Wu J, Lee K, Gao B, You M: The detrimental role played by lipocalin-2 in alcoholic fatty liver in mice. Am J Pathol 2016, 186:2417-2428

18. Wieser V, Tymoszuk P, Adolph TE, Grander C, Grabherr F, Enrich B, Pfister A, Lichtmanegger L, Gerner R, Drach M, Moser P, Zoller H, Weiss G, Moschen AR, Theurl I, Tilg H: Lipocalin 2 drives neutrophilic inflammation in alcoholic liver disease. J Hepatol 2016, 64:872-880

19. Hu M, Yin H, Mitra MS, Liang X, Ajmo JM, Nadra K, Chrast R, Finck BN, You M: Hepatic-specific lipin-1 deficiency exacerbates experimental alcohol-induced steatohepatitis in mice. Hepatology 2013, 58:1953-1963

20. Jiang Z, Zhou J, Zhou D, Zhu Z, Sun L, Nanji AA: The adiponectinSIRT1-AMPK pathway in alcoholic fatty liver disease in the rat. Alcohol Clin Exp Res 2015, 39:424-433

21. Stockwell BR, Friedmann Angeli JP, Bayir H, Bush AI, Conrad M, Dixon SJ, Fulda S, Gascón S, Hatzios SK, Kagan VE, Noel K, Jiang X, Linkermann A, Murphy ME, Overholtzer M, Oyagi A, Pagnussat GC, Park J, Ran Q, Rosenfeld CS, Salnikow K, Tang D, Torti FM, Torti SV, Toyokuni S, Woerpel KA, Zhang DD: Ferroptosis: a regulated cell death nexus linking metabolism, redox biology, and disease. Cell 2017, 171:273-285

22. Wang H, An P, Xie E, Wu Q, Fang X, Gao H, Zhang Z, Li Y, Wang X, Zhang J, Li G, Yang L, Liu W, Min J, Wang F: Characterization of ferroptosis in murine models of hemochromatosis. Hepatology 2017, 66:449-465

23. Shimada K, Hayano M, Pagano NC, Stockwell BR: Cell-line selectivity improves the predictive power of pharmacogenomic analyses and helps identify NADPH as biomarker for ferroptosis sensitivity. Cell Chem Biol 2016, 23:225-235

24. Yao X, Zhang Y, Hao J, Duan HQ, Zhao CX, Sun C, Li B, Fan BY, Wang X, Li WX, Fu XH, Hu Y, Liu C, Kong XH, Feng SQ: Deferoxamine promotes recovery of traumatic spinal cord injury by inhibiting ferroptosis. Neural Regen Res 2019, 14:532-541

25. Chen MS, Wang SF, Hsu CY, Yin PH, Yeh TS, Lee HC, Tseng LM: CHAC1 degradation of glutathione enhances cystine-starvationinduced necroptosis and ferroptosis in human triple negative breast cancer cells via the GCN2-eIF2alpha-ATF4 pathway. Oncotarget 2017, 8:114588-114602

26. Kang R, Kroemer G, Tang D: The tumor suppressor protein p53 and the ferroptosis network. Free Radic Biol Med 2019, 133:162-168 
27. Louandre C, Ezzoukhry Z, Godin C, Barbare JC, Mazière JC, Chauffert B, Galmiche A: Iron-dependent cell death of hepatocellular carcinoma cells exposed to sorafenib. Int J Cancer 2013, 133:1732-1742

28. Jennis M, Kung CP, Basu S, Budina-Kolomets A, Leu JI, Khaku S, Scott JP, Cai KQ, Campbell MR, Porter DK, Wang X, Bell DA, Li X, Garlick DS, Liu Q, Hollstein M, George DL, Murphy ME: An African-specific polymorphism in the TP53 gene impairs p53 tumor suppressor function in a mouse model. Genes Dev 2016, 30:918-930

29. Jiang L, Kon N, Li T, Wang SJ, Su T, Hibshoosh H, Baer R, Gu W: Ferroptosis as a p53-mediated activity during tumour suppression. Nature 2015, 520:57-62

30. Wang SJ, Li D, Ou Y, Jiang L, Chen Y, Zhao Y, Gu W: Acetylation is crucial for p53-mediated ferroptosis and tumor suppression. Cell Rep 2016, 17:366-373

31. Hayano M, Yang WS, Corn CK, Pagano NC, Stockwell BR: Loss of cysteinyl-tRNA synthetase (CARS) induces the transsulfuration pathway and inhibits ferroptosis induced by cystine deprivation. Cell Death Differ 2016, 23:270-278

32. Luo $\mathrm{M}$, Wu L, Zhang $\mathrm{K}$, Wang $\mathrm{H}$, Zhang $\mathrm{T}$, Gutierrez $\mathrm{L}$, O'Connell D, Zhang P, Li Y, Gao T, Ren W, Yang Y: miR-137 regulates ferroptosis by targeting glutamine transporter SLC1A5 in melanoma. Cell Death Differ 2018, 25:1457-1472

33. Li Y, Feng D, Wang Z, Zhao Y, Sun R, Tian D, Liu D, Zhang F, Ning S, Yao J, Tian X: Ischemia-induced ACSL4 activation contributes to ferroptosis-mediated tissue injury in intestinal ischemia/reperfusion. Cell Death Differ 2019, 26:2284-2299

34. Hou W, Xie Y, Song X, Sun X, Lotze MT, Zeh HJ 3rd, Kang R, Tang D: Autophagy promotes ferroptosis by degradation of ferritin. Autophagy 2016, 12:1425-1428

35. Devos D, Moreau C, Kyheng M, Garçon G, Rolland AS, Blasco H, Gelé P, Timothée Lenglet T, Veyrat-Durebex C, Corcia P, Dutheil M, Bede P, Jeromin A, Oeckl P, Otto M, Meninger V, Danel-Brunaud V, Devedjian JC, Duce JA, Pradat PF: A ferroptosis-based panel of prognostic biomarkers for amyotrophic lateral sclerosis. Sci Rep 2019, 9:2918

36. Zhang Z, Yao Z, Wang L, Ding H, Shao J, Chen A, Zhang F, Zheng S: Activation of ferritinophagy is required for the RNA- binding protein ELAVL1/HuR to regulate ferroptosis in hepatic stellate cells. Autophagy 2018, 14:2083-2103

37. Wang J, Kainrad N, Shen H, Zhou Z, Rote P, Zhang Y, Nagy LE, Wu J, You M: Hepatic knockdown of splicing regulator Slu7 ameliorates inflammation and attenuates liver injury in ethanol-fed mice. Am J Pathol 2018, 188:1807-1819

38. Mittler R, Darash-Yahana M, Sohn YS, Bai F, Song L, Cabantchik IZ, Jennings PA, Onuchic JN, Nechushtai R: NEET proteins: a new link between iron metabolism, reactive oxygen species, and cancer. Antioxid Redox Signal 2019, 30:1083-1095

39. Yuan H, Li X, Zhang X, Kang R, Tang D: CISD1 inhibits ferroptosis by protection against mitochondrial lipid peroxidation. Biochem Biophys Res Commun 2016, 478:838-844

40. Kim EH, Shin D, Lee J, Jung AR, Roh JL: CISD2 inhibition overcomes resistance to sulfasalazine-induced ferroptotic cell death in head and neck cancer. Cancer Lett 2018, 432:180-190

41. Song E, Ramos SV, Huang X, Liu Y, Botta A, Sung HK, Turnbull PC, Wheeler MB, Berger T, Wilson DJ, Perry CGR, Mak TW, Sweeney G: Holo-lipocalin-2-derived siderophores increase mitochondrial ROS and impair oxidative phosphorylation in rat cardiomyocytes. Proc Natl Acad Sci U S A 2018, 115:1576-1581

42. Li W, Feng G, Gauthier JM, Lokshina I, Higashikubo R, Evans S, Liu X, Hassan A, Tanaka S, Cicka M, Hsiao HM, Ruiz-Perez D, Bredemeyer A, Gross RW, Mann DL, Tyurina YY, Gelman AE, Kagan VE, Linkermann A, Lavine KJ, Kreisel D: Ferroptotic cell death and TLR4/Trif signaling initiate neutrophil recruitment after heart transplantation. J Clin Invest 2019, 129:2293-2304

43. Singh V, Yeoh BS, Chassaing B, Zhang B, Saha P, Xiao X, Awasthi D, Shashidharamurthy R, Dikshit M, Gewirtz A, VijayKumar M: Microbiota-inducible innate immune, siderophore binding protein lipocalin 2 is critical for intestinal homeostasis. Cell Mol Gastroenterol Hepatol 2016, 2:482-498.e6

44. Sano T, Huang W, Hall JA, Yang Y, Chen A, Gavzy SJ, Lee JY, Ziel JW, Miraldi ER, Domingos AI, Bonneau R, Littman DR: An IL23R/IL-22 circuit regulates epithelial serum amyloid A to promote local effector Th17 responses. Cell 2015, 163:381-393 